\title{
Dacryocystorhinostomy for the treatment of nasolacrimal duct obstruction: a pilot trial to assess impact on coexisting sinus symptoms
}

\author{
Ankur Mehta ${ }^{1,3}$, Wan $\mathrm{Yi}^{1}$, Arjuna Ananda ${ }^{2}$, Raf Ghabrial ${ }^{1-3}$ \\ ${ }^{1}$ Sydney Eye Hospital, Sydney, Australia; ${ }^{2}$ Royal Prince Alfred Hospital, Sydney, \\ Australia; ${ }^{3}$ University of Sydney Medical School, Sydney, Australia
}

\begin{abstract}
Purpose: To report on coexistent sinus symptoms in a population of patients who underwent endonasal dacryocystorhinostomy (DCR) surgery for nasolacrimal duct obstruction (NLDO) in a pilot study designed to test and refine methods and to estimate patient sample size for a larger multicentre randomised trial.

Methods: Ninety-four consecutive patients with NLDO who underwent endonasal $D C R$ by three surgeons in both public and private practice over a two-year period were included in this study. Questionnaires were given preceding DCR surgery and at 10 weeks post-surgery. Sinus symptoms including sinusitis, nasal congestion, hyposmia, nasal discharge, and facial pressure were assessed in the questionnaire. All subjects underwent primary endonasal DCR and all patients underwent the same post-operative regimen. Results: Questionnaire responses revealed that 48/94 (51\%) patients had one or more sinus symptoms prior to DCR surgery. Ten-week post-DCR follow-up questionnaires were obtained from $77 / 94$ (82\%) patients. About 20/31 (65\%) patients with one or more sinus symptoms prior to DCR surgery reported resolution of coexisting sinus symptoms by 10 weeks post-DCR surgery.

Conclusion: The data from this pilot study suggest that approximately half of patients attending for NLDO have coexisting sinus symptoms. There was some loss to follow-up. This pilot study highlighted the need for a validated patient questionnaire, longer length of follow-up, control groups for surgical intervention, and use of perioperative medications. Any clinical trial designed to assess the impact of endonasal surgery on sinus symptoms would need to enrol several hundred patients in order to reach a statistically valid conclusion.
\end{abstract}

Keywords: dacryocystorhinostomy, nasolacrimal duct obstruction, sinus symptoms

\section{Introduction}

Nasolacrimal duct obstruction (NLDO) can cause epiphora, blurred vision, and recurrent superficial ocular infections. ${ }^{1}$ Dacryocystorhinostomy (DCR) is the recognised standard surgical treatment for nasolacrimal obstruction. ${ }^{1,2}$ External

Correspondence: Ankur Mehta, Sunder Eye Infirmary, Queen Alexander Rd,

Sunderland SR2 9HP, UK.

E-mail: drankurmehta@outlook.com 
or endonasal approaches for DCR are accepted as valid treatments for NLDO. ${ }^{2}$ Our clinical experience has shown that patients attending the clinic with symptoms of NLDO have reported coexisting sinus symptoms. It has been shown that up to $70 \%$ of patients with NLDO have radiological evidence of sinus pathology ${ }^{3}$, but there is a paucity of data reporting the subjective impact of sinus disease on patients presenting with NLDO.

Patient satisfaction and symptom relief are key outcome measures when assessing the success of surgical intervention. Patient questionnaires have been shown to be useful tools in measuring these outcomes. ${ }^{1,4}$ The authors designed a questionnaire-based prospective pilot study to investigate the relationship between NLDO and sinus disease and to evaluate if surgical intervention for NLDO had an impact on the reported sinus symptoms.

\section{Materials and methods}

This prospective qualitative pilot study was made up of 94 participants from both private practice and quaternary referral hospital clinics. Three surgeons performed DCR surgery at three separate locations. All participants underwent primary endonasal DCR for NLDO. Surgical technique was similar between the three participating surgeons:

1. Incision in the lateral mucosa at the ridge above the base of the inferior turbinate, just lateral to the anterior lip of the middle turbinate at the level of the lacrimal sac, using a no. 11 blade.

2. Kerrison Rongeur was used to create the osteotomy.

3. Punctal probe inserted in inferior puncta and advanced into lacrimal sac.

4. Probe position used to identify lacrimal sac and visualised endonasally.

5. Incision and flap formation of lacrimal sac. Flaps reflected into nasal cavity opening.

6. Silicone tubal stents were inserted from the superior and inferior puncta and joined (by knotting) in the nasal cavity.

7. Stat triamcinolone $(40 \mathrm{mg} / \mathrm{ml}$ in $1 \mathrm{ml}$ preparation) was used post-stent insertion (one drop at the site of puncta).

8. The participants were advised to use topical chloramphenicol ( $1 \%$ in a $4 \mathrm{~g}$ preparation) ointment or drops for three days post-procedure.

9. Silicone tubal stents were removed at six weeks in an outpatient appointment.

A questionnaire was provided to all participants (Appendix 1). The questionnaire was based on the most common symptoms of sinus pathology. Participants were asked about previous history of sinusitis and previous sinus surgery. Specific sinus symptom history was recorded with regards to the following: nasal congestion, 
post-nasal drip, nasal discharge, hyposmia, and facial pressure. Participants completed an identical questionnaire at 10 weeks post-surgery in a follow-up appointment. Patients who had initially reported coexisting sinus symptoms formed the cohort for evaluating any impact of DCR surgery.

Written informed consent was obtained from all participants. Ethics approval was obtained from the Human Research Ethics Committee-Northern Sector of the South Eastern Sydney Local Health District of NSW Health, Australia. All procedures performed in studies involving human participants were in accordance with the ethical standards of the institutional and/or national research committee and with the 1964 Helsinki Declaration and its later amendments or comparable ethical standards.

\section{Results}

Ninety-four subjects were recruited for prospective analysis prior to DCR surgery. Sixty two were female (66\%). Forty-eight participants (51\%) comprising 31 females $(65 \%)$ reported one or more symptoms of sinusitis prior to DCR surgery. Some participants reported more than one sinus symptom.

The reported coexisting symptom was nasal congestion (35\%), facial pressure (16\%), nasal discharge (10\%), and hyposmia (7\%). Seventeen of these participants (18\%) were lost to follow-up at 10 weeks (Table 1). By comparing the pre-surgery questionnaire with the post-operative questionnaire, a score of "improvement" was given if the symptom documented in the pre-operative questionnaire was omitted from the post-operative questionnaire. In cases where more than one sinus symptom was documented, scoring "improvement" was given if one of the sinus symptoms was omitted from the post-operative questionnaire. Of the 31 remaining participants who had reported pre-existing sinus symptoms, 20 (65\%) reported improvement in their sinus symptoms following DCR surgery (Fig. 1).

Of the 46 participants who reported no symptoms of sinusitis prior to DCR surgery, none were lost to follow-up. Of these participants, no one reported onset of symptoms of sinusitis at 10-week follow-up.

Table 1. Sinus symptoms reported

\begin{tabular}{|l|c|c|}
\hline Sinus symptoms & Totals & Total (\%) \\
\hline Nasal congestion & 33 & 35 \\
\hline Facial pressure & 15 & 16 \\
\hline Nasal discharge & 9 & 10 \\
\hline Hyposmia & 7 & 7 \\
\hline
\end{tabular}




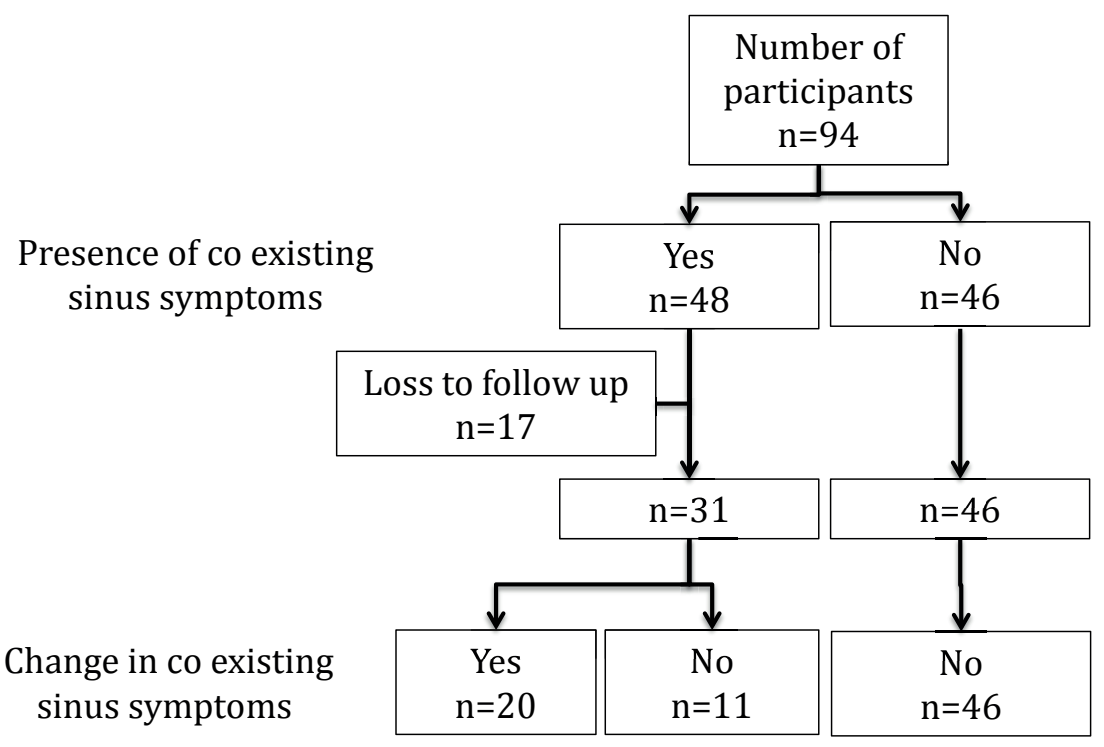

Fig. 1. Study design and outcomes.

\section{Discussion}

There is a paucity of data in the literature evaluating the relationship between the symptoms associated with sinus disease and the symptoms associated with NLDO. The aim of this pilot study was to identify if a relationship does exist and to give direction for further studies to evaluate this relationship. In this study, we found that $51 \%$ of patients who attended for endonasal DCR surgery for treatment of NLDO reported coexisting sinus symptoms. A study performed by Eyigor et al. reviewed 37 patients undergoing DCR surgery for NLDO. Paranasal computed tomography imaging showed that $73.3 \%$ had objective sinonasal abnormalities consistent with sinus disease. This suggests that there may be poor correlation between objective sinus disease (73.3\%) and subjective symptoms of sinus disease (51\%). Interestingly, the study reported a positive correlation between radiological evidence of sinus disease and NLDO and a lower success rate in treating NLDO with endonasal DCR in the cohort that had radiological evidence of sinus disease, and this may suggest a possible relationship between the two pathologies. ${ }^{3}$

The questionnaire used in this study was based on the common sinusrelated symptoms (Appendix 1). Smirnov et al. trialled a novel scoring system to evaluate NLDO and endonasal DCR called the NLDO Symptom Score (NLDO-SS). ${ }^{1}$ 
Sixty-four patients were reviewed and their subjective responses were compared with a standardised, validated generic item measure of patient benefit called the Glasgow Benefit Inventory (GBI). They concluded that endonasal DCR improved quality of life when measured using the $G B I$ and that their questionnaire correlated well with the GBI score and gave more information about the benefits after endonasal DCR than GBI alone. ${ }^{1}$ Penttila et al. validated the NLDO-SS. They reviewed 86 eyes that underwent endonasal DCR and compared an objective outcome measure (irrigation of nasolacrimal apparatus) with the NLDO-SS qualitative outcome. ${ }^{4}$ They reported that the NLDO-SS was a feasible clinical tool in measuring outcomes for endonasal DCR. The NLDO-SS asked for responses related to NLDO: tearing, discharge, swelling around the eyes, pain around the eyes, change in visual acuity, and included two further categories related to nasal symptoms: nose blockage and nasal cavity discharge. These criteria are similar to the questionnaire used in this study. The use of a validated questionnaire-based qualitative tool like the NLDO-SS would improve the interpretation of outcomes.

In this study, a standard 10-week follow-up was used to assess the outcome of endonasal DCR procedure. Solar et al. assessed 275 subjects by questionnaire at $3,6,12$, and 18 months post-surgery and found that peak improvement was reported at 3 months ${ }^{5}$, and Young et al. ${ }^{6}$ reviewed 82 patients undergoing endoscopic sinus surgery for chronic sinusitis. In this group, a visual analogue score (VAS) was used to assess symptoms. The score was repeated at 3, 6, 12, 24, and 36 months. Analysis showed that the VAS score at 3 months was not statistically different to that VAS score at 36 months. This suggests that the optimal time to assess sinus symptoms post-surgery is at three months or 12 weeks. The two-week variation in assessment is a potential bias in this study, and its impact on the outcome is difficult to quantify.

In this study, we showed that $65 \%$ of patients who reported sinus symptoms pre-DCR reported improvement in their symptoms post-endonasal DCR. This may indicate a relationship between NLDO and sinus disease. The mechanism by which endonasal DCR may impact sinus disease is unclear.

Endonasal DCR uses instrumentation in the nasal cavity and intraoperative and post-operative medications. This raises the question if the surgical procedure itself or the medications used improves symptoms by changing the calibre of the nasal cavity or impacting in sinus disease, respectively. The authors could find no previous studies assessing the calibre of the nasal cavity pre- or post-endonasal sinus or DCR surgery. To assess for the impact of medications, a trial of the regimen used intra- and post-operatively on subjects presenting for NLDO surgery with coexisting sinus disease pre-surgery and assessing outcomes using a qualitative questionnaire is one option to control for this variable. The authors recognise this as a potential bias in this study. 
The anatomical proximity of the nasolacrimal duct and the sinus system in the head and neck are well documented in ophthalmology anatomy text; ${ }_{;}^{7}$ so it is reasonable to hypothesise that a pathological disease that is affecting one system could be involving the second. ${ }^{3}$ Both structures are lined with mucosal tissue. The nasolacrimal duct is lined with goblet cells and intraepithelial mucus glands and has microvilli present; ${ }^{8}$ the sinus systems are also lined with goblet cells but have ciliated pseudo-stratified epithelium cells instead; and these similar histopathological features reflect the similar defensive functions of the two structures.

Approximately half of the lymphocytes of the immune system are in mucosa-associated lymphoid tissue (MALT). ${ }^{9}$ MALT tissue is present in the nasolacrimal duct, referred to as lacrimal duct-associated lymphoid tissue, and documented MALT-associated lymphomas have been reported in the paranasal sinus. ${ }^{10}$ It has been shown that although MALT sites can be anatomically separated they remain functionally connected in what has been termed the common mucosal immune system. ${ }^{11}$ This could suggest that an inflammatory response in the nasolacrimal duct could stimulate a similar response in the sinus system or vice versa, but in the absence of any evidence this remains purely speculative. Shams et al. reviewed 196 patients undergoing DCR. ${ }^{12}$ Of the cohort, 20 subjects (10.2\%) had chronic rhinosinusitis (CRS) and they found three of these (1.5\%) developed acute rhinosinusitis (ARS), although all three were symptom free at the time of surgery. The authors suggest that CRS is a risk factor for developing ARS post-DCR. In their review, the authors discount surgical trauma or stent insertion as a cause for ARS (video review of surgical procedure and all three cases did not have stents inserted) and felt that perhaps either acquired or congenital narrow nasal space or nasociliary dysfunction secondary to CRS may have attributed to developing ARS. The mechanism for ARS post-DCR is unclear.

A limitation of our study was the loss to follow-up for 17 participants who had reported one or more sinus symptoms preceding DCR surgery.

\section{Conclusion}

These results indicate that a significant proportion of patients who attend for DCR surgery for the treatment of NLDO have coexisting subjective sinus symptoms. In this pilot study, we showed that the treatment of NLDO with endonasal DCR improved coexisting sinus symptoms in $65 \%$ of these patients. In this study, we found that a sample size greater that 385 would be needed in any clinical trial designed to evaluate the role of endonasal DCR surgery for NLDO on sinus disease to reach a statistically valid conclusion. This pilot study highlights the need for further studies in this area to further explore the relationship between sinus symptoms and NLDO and the potential treatment outcomes of endonasal DCR on coexisting sinus symptoms. 


\section{References}

1. Smirnov $\mathrm{G}$, Tuomilehto $\mathrm{H}$, Kokki $\mathrm{H}$, et al. Symptom score questionnaire for nasolacrimal duct obstruction in adults-a novel tool to assess the outcome after endoscopic dacryocystorhinostomy. Rhinology. 2010;48(4):446-451.

2. Leong SC, Macewen CJ, White PS. A systematic review of outcomes after dacryocystorhinostomy in adults. Am J Rhinol Allergy. 2010;24(1):81-90.

3. Eyigor $\mathrm{H}$, Unsal Al, Unsal A. The role of accompanying sinonasal abnormalities in the outcome of endonasal dacryocystorhinostomy. Am J Rhinol. 2006;20(6):620-624.

4. Penttila E, Smirnov G, Seppa J, Tuomilehto H, Kokki H. Validation of a symptom-score questionnaire and long-term results of endoscopic dacryocystorhinostomy. Rhinology. 2014;52(1):84-89.

5. Soler ZM, Mace J, Smith TL. Symptom-based presentation of chronic rhinosinusitis and symptom-specific outcomes after endoscopic sinus surgery. Am J Rhinol. 2008;22:297-301.

6. Young J, Frenkiel S, Tewfik MA, Mouadeb DA. Long-term outcome analysis of endoscopic sinus surgery for chronic sinusitis. Am J Rhinol. 2007;21:743-747.

7. Snell RS, Lemp MA. Clinical Anatomy of the Eye. Oxford: Blackwell Scientific Publications; 1989.

8. Paulsen F. The human nasolacrimal ducts. Adv Anat Embryol Cell Biol. 2003;170:III-XI, 1-106.

9. Croitoru K, Bienenstock J. Characteristics and functions of mucosa-associated lymphoid tissue. In: P. L. Ogra JM, Lamm ME, Strober W, McGhee JR, Bienenstock J (Eds), Handbook of Mucosal Immunology. San Diego: Academic Press; 1994:141-151.

10. Tauber S, Nerlich A, Lang S. MALT lymphoma of the paranasal sinuses and the hard palate: report of two cases and review of the literature. Eur Arch Otorhinolaryngol. 2006;263(1):19-22.

11. Cesta M. Normal structure, function, and histopathology of mucosa associated lymphoid tissue. Tocicol Pathol. 2006;34:599-608.

12. Shams PN, Selva D. Acute post-operative rhinosinusitis following endonasal dacryocystorhinostomy. Eye. 2013;27(10):1130-1136. 


\section{PATIENT NAME:}

DOB:

PROCEDURE: Dacryocystorhynostomy OPEN/ENDOSCOPIC (Please circle) LEFT/RIGHT (Please circle)

SYMPTOMS: Epiphora/Dacryocystitis

\section{DATE OF PROCEDURE:}

\section{DATE OF FOLLOW-UP:}

\section{Previous history of sinusitis Yes/No (Please circle)}

If yes, did sinusitis PRECEDE/CONTINUE/COME AFTER epiphora?

\begin{tabular}{|l|c|}
\hline 2. Previous sinus surgery & $\begin{array}{c}\text { Yes/No (Please circle) } \\
\text { LEFT/RIGHT (Please circle) }\end{array}$ \\
\hline
\end{tabular}

If YES to Question 1 \&/or 2, answer the following questions:

\begin{tabular}{|c|c|c|}
\hline & PRE-PROCEDURE & AT FOLLOW-UP (10 weeks) \\
\hline 3. Nasal congestion & Yes/No & Yes/No \\
\hline 4. Post-nasal drip & Yes/No & Yes/No \\
\hline 5. Nasal discharge & Yes/No & Yes/No \\
\hline 6. Hyposmia & Yes/No & Yes/No \\
\hline \multirow[t]{4}{*}{ 7. Facial pressure } & Yes/No & Yes/No \\
\hline & \multicolumn{2}{|c|}{$\begin{array}{l}\text { If YES, state location e.g. forehead, nasal or } \\
\text { maxillary region: }\end{array}$} \\
\hline & \multicolumn{2}{|l|}{ If YES: } \\
\hline & LEFT/RIGHT & LEFT/RIGHT \\
\hline
\end{tabular}


DCR for the treatment of NLDO

\begin{tabular}{|l|c|c|}
\hline \multirow{2}{*}{ 8. Epiphora } & \multicolumn{1}{|c|}{ Yes/No } & Yes/No \\
\cline { 2 - 3 } & If YES: & \\
\cline { 2 - 3 } & LEFT/RIGHT & LEFT/RIGHT \\
\hline $\begin{array}{l}\text { 9. Regional symptoms } \\
\text { e.g. cough, irritation, } \\
\text { discomfort, change in taste, other } \\
\text { symptoms }\end{array}$ & & \\
\hline $\begin{array}{l}\text { ANY OTHER COMMENTS } \\
\text { e.g. Peri-operative complications }\end{array}$ & & \\
\hline
\end{tabular}

